\title{
Trastornos de Personalidad en Hombres Maltratadores a la Pareja: Perfil Diferencial entre Agresores en Prisión y Agresores con Suspensión de Condena
}

\section{Personality Disorders in Batterer Men: Differential Profile Between Aggressors in Prison and Aggressors with a Suspended Sentence}

José Antonio Echauri Tijeras

PSIMAE Instituto de Psicología Jurídica y Forense, España María A. Martínez Sarasa

PSIMAE Instituto de Psicología Jurídica y Forense, España

\author{
Javier Fernández-Montalvo \\ Universidad Pública de Navarra, España \\ Juana M. Azcárate Seminario \\ PSIMAE Instituto de Psicología Jurídica y Forense, España
}

Resumen. En este artículo se lleva a cabo una descripción de los trastornos de personalidad que aparecen con mayor frecuencia en una muestra de 217 hombres maltratadores. Todos ellos forman parte de un programa de tratamiento especializado que es desarrollado por PSIMAE Instituto de Psicología Jurídica y Forense, y dirigido por el Servicio Social de Justicia del Gobierno de Navarra. Todos los participantes completaron el MCMI-II en el transcurso de la evaluación pretratamiento. Asimismo, se llevo a cabo una comparación en las variables de personalidad estudiadas entre los agresores con suspensión de condena $(\mathrm{N}=137)$ y aquellos que cumplían sentencia en prisión $(\mathrm{N}=80)$. Los resultados mostraron que el 79,3\% de la muestra de agresores presentaba al menos un trastorno de personalidad. El trastorno de mayor prevalencia era el trastorno obsesivo-compulsivo de la personalidad $(61.3 \%$ de los casos), seguido del trastorno paranoide (30\%). Además, los resultados mostraron la existencia de numerosas diferencias significativas entre los dos grupos estudiados. En general, los maltratadores en prisión mostraban un perfil de personalidad más grave y con más trastornos que los maltratadores que acudían a tratamiento por suspensión de condena. Se comentan las implicaciones de este estudio para la práctica clínica y para las investigaciones futuras.

Palabras clave: maltratadores, prisión, suspensión de condena, trastornos de personalidad, violencia de género.

Abstract. In this paper a study of personality disorders most frequently related to 217 male batterers is carried out. All of them form part of a specialized psychological treatment programme for gender violence, which is developed by PSIMAE Institute of Forensic and Legal Psychology, belonging to the Social Service of Justice, Government of Navarra (Spain). All the participants were assessed with the MCMI-II before beginning the treatment programme. Furthermore, a comparison of all the personality variables studied among the subjects referred by the court to the treatment programme (137 males with a suspended sentence) and those who were imprisoned $(\mathrm{n}=80)$ was conducted. According to the results, $79.3 \%$ of the sample showed at least one personality disorder. The most prevalent one was 
the obsessive-compulsive personality disorder (61.3\% of cases), followed by the paranoid personality disorder (30\%) and the dependent personality disorder (28.1\% of cases). Moreover, the results showed the existence of numerous statistically significant differences between groups. In general, imprisoned batterers showed a higher severity in personality variables than those who were referred by the court to the treatment programme. Implications of these results for further research and clinical practice are also commented. Keywords: batterers, gender violence, personality disorders, prison, suspended sentence.

\section{Introducción}

En los últimos años se ha producido en nuestro país un aumento considerable de las investigaciones sobre el perfil de los hombres que agreden física y/o psicológicamente a la mujer. Estos estudios se han llevado a cabo fundamentalmente con maltratadores que continúan conviviendo con la víctima (Fernández-Montalvo y Echeburúa, 1997) o que se encuentran en prisión cumpliendo una condena por haber cometido un delito específico y grave de violencia de género (Echeburúa, Fernández-Montalvo, y Amor, 2003; Fernández-Montalvo y Echeburúa, 2005; Fernández-Montalvo, Echeburúa, y Amor, 2005). Asimismo, recientemente se han comenzado a publicar algunos estudios con muestras de agresores que acuden a los programas de tratamiento por orden judicial, tras haber sido aplicada una suspensión de la ejecución de la pena a cambio de recibir una intervención especializada (Boira y Jodrá, 2010; Redondo, Graña, y González, 2009).

Los resultados obtenidos hasta la fecha muestran una gran dificultad para describir un perfil psicológico homogéneo entre los maltratadores. La violencia de género tiene una explicación multicausal $\mathrm{y}$, por lo tanto, los agresores no presentan unas características sociodemográficas, psicopatológicas o de personalidad homogéneas, que sean fácilmente identificables, y que permitan prevenir la aparición de episodios violentos (Fernández-Montalvo, 2009). No obstante, al margen de todo ello, hoy en día se cuenta con distintos estudios que arrojan algunas características básicas frecuentemente presentes entre estos agresores. Los maltratadores a la mujer en el hogar suelen presentar carencias psicológicas significativas, como sesgos cognitivos (pensamientos distorsionados sobre los roles sexuales y la inferioridad de la mujer, principalmente), dificultades de comunicación, irritabilidad y una falta de control de los impulsos, así como otras dificultades específicas (abuso de alcohol y celos patológicos) (Echeburúa, et al., 2003; Fernández-Montalvo y Echeburúa, 1997; Fernández-Montalvo, et al., 2005).

Por lo que se refiere a la personalidad, las investigaciones llevadas a cabo hasta la fecha muestran también una gran heterogeneidad en las características de personalidad presentes en este tipo de sujetos. Se han identificado algunos trastornos de personalidad en la población de agresores (Dinwiddie, 1992), sobre todo en aquellos que se encuentran en prisión (FernándezMontalvo y Echeburúa, 2008; Nicholls, Roesch, Olley, Ogloff, y Hemphill, 2005). Así, los más frecuentemente descritos han sido el trastorno antisocial de la personalidad, el límite y el narcisista (Hamberger y Hastings, 1991; Huss y Langhin-richsen-Rohling, 2000). En concreto, se estima que los maltratadores antisociales constituyen aproximadamente el 25\% de las muestras de agresores, o incluso más si los datos provienen de agresores que reciben un tratamiento por orden judicial (Hart, Dutton, y Newlove, 1993). Este tipo concreto de agresores presenta una violencia más generalizada -que va más allá de su pareja afectiva-, tiene un mayor historial de antecedentes delictivos y es más probable que se vea afectado por trastornos mentales (abuso o dependencia de sustancias, multiimpulsividad, etc.) Además, suelen presentar actitudes hostiles hacia las mujeres y una baja capacidad empática hacia las mismas (Huss y Langhinrichsen-Rohling, 2000; Quinsey, Harris, Rice, y Cormier, 1998; White y Gondolf, 2000).

En consonancia con este perfil más antisocial de algunos tipos de agresores, recientemente se ha comenzado a estudiar la presencia de características psicopáticas en algunos grupos de maltratadores. Los datos más recientes arrojan cifras cercanas a un $12 \%$ de sujetos con rasgos psicopáticos manifiestos (Echeburúa y Fernández-Montalvo, 2007). Sin embargo, si bien la relación existente entre la presencia de conductas violentas y la sintomatología psicopática está bien asentada en la bibliografía (Hare, 
2001), la importancia de la psicopatía en la violencia contra la pareja no está todavía bien establecida y son pocos los datos disponibles en la actualidad.

En cualquier caso, a pesar de los datos presentados, tras la publicación de la Ley de Medidas de Protección Integral contra la Violencia de Género en el año 2004 se ha producido un aumento espectacular de los programas de tratamiento para hombres maltratadores en nuestro país. La Ley establece que las Comunidades Autónomas implanten en sus respectivos territorios programas terapéuticos y rehabilitadores para sujetos que hayan sido juzgados y condenados por un delito de violencia de género. Se trata de que, en aquellos casos en los que el agresor no tenga antecedentes penales, y cuya condena sea menor de dos años, el juez pueda llevar a cabo una suspensión de la ejecución de la condena, condicionándola, entre otras cosas, a realizar dichos programas terapéuticos (Ley Orgánica 1/2004 de 28 de diciembre). Ello ha supuesto un cambio en el panorama de los programas de tratamiento de agresores, creándose propuestas específicas y variadas, incluso con características muy diferentes entre sí, para atender a este tipo concreto de población.

Desde un punto de vista legal, la decisión que toma el juez en relación con el maltratador en los casos de violencia de género obedece, fundamentalmente, a la gravedad de la conducta desarrollada por los agresores, así como a la gravedad de la sentencia impuesta. No existen, habitualmente, criterios psicológicos para decidir si el mejor lugar para la reinserción de un agresor de este tipo es acudir a un programa de intervención tras la suspensión de condena o a un programa de intervención dentro de prisión. La gravedad del delito y la severidad de la condena impuesta deciden en este sentido. Qué duda cabe que, además de razones judiciales, es importante tener también criterios psicológicos adecuados para evaluar la conveniencia de comenzar un programa de tratamiento como alternativa al ingreso en prisión. Sin embargo, hoy por hoy se desconoce el perfil más adecuado de los agresores que se beneficiarían más de una u otra modalidad de intervención. Por ello, el estudio del perfil diferencial entre los agresores que están hoy en día en prisión por un delito de violencia de género y aquellos que forman parte de los programas de tratamiento tras una suspensión de condena puede contribuir a este campo.
El presente estudio tiene como objetivo comparar las características de personalidad de los agresores condenados a una pena de prisión por un delito de violencia de género con las de aquellos agresores que acuden al programa de tratamiento como alternativa al cumplimiento de la pena en prisión (suspensión de condena). Se trata de conocer el perfil específico en cuanto a trastornos de personalidad de estos dos tipos de agresores. Este objetivo es relevante debido a la ausencia de estudios en este sentido. La hipótesis de partida es que los maltratadores en prisión presentan un perfil más grave de personalidad, con puntuaciones superiores a los que acuden al programa ambulatorio de suspensión de condena. Se esto es así, en una fase posterior se deben diseñar programas de tratamiento específicos y adaptados a las características de estos sujetos.

\section{Método}

\section{Participantes}

La muestra total de este estudio está compuesta por 217 hombres que han acudido al programa terapéutico para hombres maltratadores que se desarrolla en Navarra. Se trata de un programa de intervención con hombres violentos que lleva en funcionamiento desde el año 2005, que está desarrollado por PSIMAE Instituto de Psicología Jurídica y Forense, y es dirigido por el Servicio Social de Justicia del Gobierno de Navarra. Todos los pacientes de este estudio han sido atendidos en el período comprendido entre enero de 2005 y abril de 2009.

Las vías de acceso de los agresores al programa en el presente estudio son dos: a) el programa en régimen ambulatorio, en el que una parte de los sujetos acuden a través de suspensión de condena a recibir tratamiento $(n=137) ; y$ b) el programa en prisión, en el que maltratadores condenados a una pena de prisión se someten voluntariamente a tratamiento $(n=80)$.

En la selección de la muestra se han tenido en cuenta los siguientes criterios de admisión: a) ser un varón adulto (tener más de 18 años); b) haber ejercido algún tipo de maltrato físico o psicológico contra su pareja; c) no sufrir ningún trastorno mental grave ni enfermedad física invalidante; y d) participar 
voluntariamente en la investigación, una vez debidamente informado de las características de la misma.

Un resumen de las principales características sociodemográficas de ambas muestras se presenta en la tabla 1. La edad media de la muestra total es de 36,6 años (con una desviación típica de 9,07 y con un rango que oscila entre los 18 y los 71 años). En cuanto a la nacionalidad, es prácticamente igual la tasa de sujetos extranjeros $(50.7 \%)$ frente a la de los nacionales $(49.3 \%)$. El nivel de instrucción de la mayoría es más bien bajo, con un predominio claro de sujetos con estudios primarios (64.5\%) y con sólo un 5.1\% de sujetos con estudios universitarios. En cuanto a la situación laboral, aunque mayoritariamente los pacientes se encuentran en activo, existe una impor- tante tasa de desempleo en la muestra $(32,3 \%)$. Igualmente, el 60,8\% de los hombres de la muestra tienen hijos en común con su pareja o expareja.

\section{Medidas de evaluación}

El Inventario Clínico Multiaxial de Millon (MCMI-II) (Millon, 1997) es un cuestionario clínico autoaplicado de evaluación de la personalidad y de distintos síndromes clínicos. Está compuesto por 175 ítems de respuesta dicotómica (verdadero/ falso), que proporcionan información sobre 10 escalas básicas de personalidad (esquizoide, fóbica, dependiente, histriónica, narcisista, antisocial, agresivo-sádica,

Tabla 1. Características sociodemográficas de la muestra

\begin{tabular}{|c|c|c|c|c|}
\hline Variables & $\begin{array}{c}\text { Muestra total } \\
\text { N = 217 } \\
\text { X (DT) }\end{array}$ & $\begin{array}{c}\text { Susp. condena } \\
\mathbf{N}=\mathbf{1 3 7} \\
\mathrm{X}(\mathrm{DT})\end{array}$ & $\begin{array}{l}\text { Prisión } \\
\text { N = 80 } \\
X \text { (DT) }\end{array}$ & $\mathbf{t}$ \\
\hline Edad & $36.68(9.07)$ & $36.33(9.04)$ & $37.28(9.16)$ & 0.74 \\
\hline Años relación pareja & $8.12(7.61)$ & $8.29(7.36)$ & $7.81(8.04)$ & 0.45 \\
\hline Variables & $\begin{array}{c}\text { Muestra total } \\
\begin{array}{c}\mathbf{N}=\mathbf{2 1 7} \\
\mathrm{N}(\%)\end{array}\end{array}$ & $\begin{array}{c}\text { Susp. condena } \\
\mathbf{N}=137 \\
\mathbf{n}(\%)\end{array}$ & $\begin{array}{c}\text { Prisión } \\
\mathbf{N}=\mathbf{8 0} \\
\text { n }(\%)\end{array}$ & $\mathrm{X}^{2}$ \\
\hline \multicolumn{5}{|l|}{ Grupos de edad } \\
\hline $\begin{array}{l}18-30 \\
31-50 \\
51-65 \\
>65\end{array}$ & $\begin{array}{c}60(27.6 \%) \\
145(66.8 \%) \\
11(5.1 \%) \\
1(0.5 \%)\end{array}$ & $\begin{array}{c}39(28.5 \%) \\
91(66.4 \%) \\
6(4.4 \%) \\
1(0.7 \%)\end{array}$ & $\begin{array}{c}21(26.3 \%) \\
54(67.5 \%) \\
5(6.2 \%) \\
--\end{array}$ & 1.03 \\
\hline \multicolumn{5}{|l|}{ Nacionalidad } \\
\hline $\begin{array}{l}\text { Español } \\
\text { Extranjero }\end{array}$ & $\begin{array}{l}107(49.3 \%) \\
110(50.7 \%)\end{array}$ & $\begin{array}{l}59(43.1 \%) \\
78(56.9 \%)\end{array}$ & $\begin{array}{l}48(60 \%) \\
32(40 \%)\end{array}$ & $5.79 *$ \\
\hline \multicolumn{5}{|l|}{ Estudios } \\
\hline $\begin{array}{l}\text { Primarios } \\
\text { Secundarios } \\
\text { Universitarios }\end{array}$ & $\begin{array}{c}140(64.5 \%) \\
66(30.4 \%) \\
11(5.1 \%)\end{array}$ & $\begin{array}{c}80(58.4 \%) \\
50(36.5 \%) \\
7(5.1 \%)\end{array}$ & $\begin{array}{c}60(75 \%) \\
16(20 \%) \\
4(5 \%)\end{array}$ & $6.68^{*}$ \\
\hline \multicolumn{5}{|l|}{ Situación laboral } \\
\hline $\begin{array}{l}\text { Activo } \\
\text { Desempleado } \\
\text { Jubilado }\end{array}$ & $\begin{array}{c}140(64.5 \%) \\
70(32.3 \%) \\
7(3.2 \%)\end{array}$ & $\begin{array}{c}97(70.8 \%) \\
36(26.3 \%) \\
4(2.9 \%)\end{array}$ & $\begin{array}{c}43(53.8 \%) \\
34(42.4 \%) \\
3(3.8 \%)\end{array}$ & $6.50^{*}$ \\
\hline \multicolumn{5}{|l|}{ Hijos en común } \\
\hline $\begin{array}{l}\text { Sí } \\
\text { No }\end{array}$ & $\begin{array}{r}132(60.8 \%) \\
85(39.2 \%)\end{array}$ & $\begin{array}{l}88(64.2 \%) \\
49(35.8 \%)\end{array}$ & $\begin{array}{l}44(55 \%) \\
36(45 \%)\end{array}$ & 1.81 \\
\hline
\end{tabular}


compulsiva, pasivo-agresiva y autodestructiva), 3 escalas de personalidad patológica (esquizoide, límite y paranoide), 6 síndromes clínicos de gravedad moderada (ansiedad, histeriforme, hipomanía, distimia, abuso de alcohol y abuso de drogas) y 3 síndromes clínicos de gravedad severa (pensamiento psicótico, depresión mayor y trastorno delirante). Debido al objetivo de este estudio, se presentan solamente los resultados de las 13 escalas de personalidad.

\section{Procedimiento}

Los sujetos de este estudio forman parte de un programa de intervención con hombres maltratadores contra la pareja desarrollado en Navarra. Una vez seleccionada la muestra clínica con arreglo a los criterios previamente señalados, todos ellos fueron evaluados por el equipo de psicólogos del programa.

En concreto, se llevaron a cabo dos sesiones de evaluación. En estas sesiones se recogieron los datos sociodemográficos y de maltrato, así como las variables relacionada con la personalidad a través del MCMI-II. Una vez completada la evaluación, todos los pacientes comenzaban el tratamiento psicológico para hombres maltratadores en el mismo centro.

Con arreglo a los criterios más conservadores de Weltzler (1990), en este estudio sólo se ha considerado la presencia de un trastorno de personalidad cuando la puntuación en la tasa-base (TB) del $M C M I-I I$ era superior a 84.

\section{Análisis estadísticos}

Los análisis estadísticos han sido llevados a cabo con el programa SPSS (versión 15.0 para Windows). Para determinar las características de la muestra se ha llevado a cabo un análisis de carácter descriptivo (porcentajes, medias y desviaciones típicas). Asimismo, la comparación entre los grupos se ha realizado mediante la prueba Chi cuadrado, en el caso de las variables categóricas, y la t de Student para las variables cuantitativas.

\section{Resultados}

A continuación se presentan los resultados referidos, en primer lugar, a las puntuaciones directas obtenidas en el MCMI-II; en segundo lugar se analizan los resultados desde una perspectiva cualitativa, considerando la existencia de un trastorno de personalidad cuando la puntuación en la tasa-base (TB) del MCMI-II era superior a 84.

\section{Resultados en las dimensiones del MCMI-II}

En la tabla 2 se presentan las puntuaciones medias obtenidas en el MCMI-II por los sujetos de la muestra, así como los resultados de la comparación entre ambos grupos.

Como se puede observar, la comparación entre

Tabla 2. Puntuación media en las escalas de trastornos de personalidad (MCMI-II)

\begin{tabular}{|c|c|c|c|c|c|c|c|}
\hline \multirow[t]{2}{*}{$\begin{array}{l}\text { Trastornos de } \\
\text { personalidad }\end{array}$} & \multicolumn{2}{|c|}{$\begin{array}{c}\text { Muestra total } \\
\mathrm{N}=\mathbf{2 1 7}\end{array}$} & \multicolumn{2}{|c|}{$\begin{array}{c}\text { Susp. condena } \\
\mathbf{N}=\mathbf{1 3 7}\end{array}$} & \multicolumn{2}{|c|}{$\begin{array}{l}\text { Prisión } \\
\mathbf{N}=80\end{array}$} & \multirow[t]{2}{*}{$t$} \\
\hline & $\bar{X}$ & (D.T.) & $\bar{X}$ & (D.T.) & $\bar{X}$ & (D.T.) & \\
\hline Esquizoide & 55.18 & $(25.77)$ & 53.58 & (25.77) & 57.93 & $(25.71)$ & 1.20 \\
\hline Fóbico & 43.15 & $(25.68)$ & 39.31 & (26.02) & 49.73 & $(23.86)$ & $2.93 * *$ \\
\hline Dependiente & 63.74 & $(28.80)$ & 61.62 & (27.89) & 67.69 & (29.99) & 1.67 \\
\hline Histriónico & 55.54 & $(25.95)$ & 52.86 & $(24.02)$ & 60.13 & (28.53) & $2.00 *$ \\
\hline Narcisista & 60.82 & $(26.87)$ & 58.01 & (27.04) & 65.67 & (26.03) & $2.03 *$ \\
\hline Antisocial & 52.32 & $(30.16)$ & 47.95 & (29.82) & 59.81 & $(29.42)$ & $2.84 * *$ \\
\hline Agresivo-sádico & 45.70 & (28.03) & 41.95 & (26.99) & 52.11 & (28.77) & $2.61 *$ \\
\hline Compulsivo & 85.37 & (28.82) & 85.18 & (27.73) & 85.71 & (29.66) & 0.13 \\
\hline Pasivo-agresivo & 39.04 & $(26.43)$ & 35.14 & (25.91) & 45.71 & (26.14) & $2.89 * *$ \\
\hline Autodestructivo & 43.17 & $(27.30)$ & 35.37 & (26.69) & 52.81 & (25.73) & $4.12 * * *$ \\
\hline Esquizotípico & 56.20 & $(31.24)$ & 50.55 & (29.92) & 65.86 & $(31.27)$ & $3.58 * * *$ \\
\hline Límite & 45.67 & $(25.03)$ & 40.47 & (23.94) & 54.58 & (24.48) & $4.15^{* * *}$ \\
\hline Paranoide & 64.56 & (32.96) & 59.83 & (32.45) & 72.65 & (32.45) & $2.81 * *$ \\
\hline
\end{tabular}

$* p<.05 ; * * p<.01 ; * * * p<.001$ 
los dos grupos estudiados muestra la existencia de diferencias significativas en la práctica totalidad de escalas del MCMI-II. Con la excepción de sólo tres escalas, en todos estos casos, los agresores que se encuentran cumpliendo una pena de prisión por haber cometido un delito de violencia de género muestran una puntuación significativamente más alta que los agresores a los que se les ha aplicado una suspensión de la ejecución de la pena.

\section{Tasa de trastornos de personalidad}

Los resultados obtenidos a través del Inventario Clínico Multiaxial de Millon (MCMI-II) en relación con los trastornos de personalidad aparecen descritos en la tabla 3. Como se puede aprecia en dicha tabla, los resultados reflejan una elevada tasa de trastornos de personalidad. Así, el 79.3\% de los sujetos de la muestra presenta al menos un trastorno de personalidad según este instrumento, por lo que únicamente un $20.7 \%$ de los sujetos no presenta ningún tipo de trastorno. Además, los 202 sujetos diagnosticados con trastornos de personalidad presentan una media de más de 3 trastornos (3.12 trastornos de personalidad por sujeto).

Por otra parte, si se analizan los resultados en función del tipo de trastorno de personalidad que presentan, los hallazgos son poco precisos. De hecho, todos los trastornos de personalidad se encuentran presentes en mayor o menor medida y todas las escalas resultan las más elevadas en al menos uno de los sujetos.

Dentro de las escalas básicas de trastornos de personalidad, el más frecuente es el trastorno compulsivo con 133 sujetos, lo que supone un $61.3 \%$ sobre el total de la muestra. A continuación, los trastornos más frecuentes son el trastorno dependiente con un $28.1 \%$ y el trastorno narcisista con un $21.2 \%$ sobre el total de la muestra.

Además, es importante señalar que, dentro de las escalas de personalidad patológica más severa y que reflejan niveles moderados o marcados de deterioro, aparece el trastorno paranoide con un $30 \%$ y el trastorno esquizotípico que se encuentra presente en el $25.3 \%$ del total de la muestra.

Cuando se comparan los resultados obtenidos en función del acceso al programa (grupo de suspensión de condena y grupo de prisión) los hallazgos son nuevamente poco precisos. Todos los trastornos de personalidad se encuentran presentes en mayor o menor medida y todas las escalas resultan las más elevadas en al menos uno de los sujetos. En el grupo de suspensión de condena el $77.4 \%$ de los sujetos presentan al menos un trastorno de personalidad frente al $82.5 \%$ de los sujetos del grupo de prisión, no habiendo diferencias significativas. Por lo tanto, un $22.6 \%$ y un $17.5 \%$ de los sujetos de cada grupo

Tabla 3. Tasa de trastornos de personalidad (MCMI-II)

\begin{tabular}{|c|c|c|c|c|c|c|c|}
\hline \multirow[t]{2}{*}{$\begin{array}{l}\text { Trastornos de } \\
\text { personalidad }\end{array}$} & \multicolumn{2}{|c|}{$\begin{array}{c}\text { Muestra total } \\
\mathbf{N}=\mathbf{2 1 7}\end{array}$} & \multicolumn{2}{|c|}{$\begin{array}{c}\text { Susp. condena } \\
\quad \mathbf{N}=137\end{array}$} & \multicolumn{2}{|c|}{$\begin{array}{l}\text { Prisión } \\
N=80\end{array}$} & \multirow[t]{2}{*}{$\mathbf{X}^{2}$} \\
\hline & $\mathbf{N}$ & $(\%)$ & $\mathbf{N}$ & $(\%)$ & $\mathbf{N}$ & $(\%)$ & \\
\hline Esquizoide & 29 & $(13.4 \%)$ & 16 & $(11.7 \%)$ & 13 & $(16.2 \%)$ & 0.91 \\
\hline Fóbico & 13 & $(6 \%)$ & 7 & $(5.1 \%)$ & 6 & $(7.5 \%)$ & 0.51 \\
\hline Dependiente & 61 & $(28.1 \%)$ & 33 & $(24.1 \%)$ & 29 & $(36.2 \%)$ & $4.15^{*}$ \\
\hline Histriónico & 35 & $(16.1 \%)$ & 15 & $(10.9 \%)$ & 20 & $(25 \%)$ & $7.37 * *$ \\
\hline Narcisista & 46 & $(21.2 \%)$ & 24 & $(17.5 \%)$ & 22 & $(27.5 \%)$ & 3.01 \\
\hline Antisocial & 37 & $(17.1 \%)$ & 20 & $(14.6 \%)$ & 17 & $(21.2 \%)$ & 1.58 \\
\hline Agresivo-sádico & 19 & $(8.8 \%)$ & 8 & $(5.8 \%)$ & 11 & $(13.7 \%)$ & $3.96^{*}$ \\
\hline Compulsivo & 133 & $(61.3 \%)$ & 82 & $(59.9 \%)$ & 51 & $(63.7 \%)$ & 0.32 \\
\hline Pasivo-agresivo & 10 & $(4.6 \%)$ & 4 & $(2.9 \%)$ & 6 & $(7.5 \%)$ & 2.41 \\
\hline Autodestructivo & 18 & $(8.3 \%)$ & 9 & $(6.6 \%)$ & 9 & $(11.2 \%)$ & 1.45 \\
\hline Esquizotípico & 55 & $(25.3 \%)$ & 27 & $(19.7 \%)$ & 28 & $(35 \%)$ & $6.24 *$ \\
\hline Límite & 15 & $(6.9 \%)$ & 5 & $(3.6 \%)$ & 10 & $(12.5 \%)$ & $6.15^{*}$ \\
\hline Paranoide & 65 & $(30 \%)$ & 33 & $(24.1 \%)$ & 32 & $(40 \%)$ & $6.10^{*}$ \\
\hline TOTAL $^{1}$ & 172 & $(79.3 \%)$ & 106 & $(77.4 \%)$ & 66 & $(82.5 \%)$ & 0.81 \\
\hline
\end{tabular}

$* p<.05 ; * * p<.01$

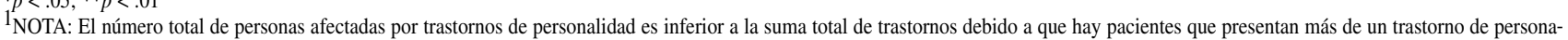
lidad. 
respectivamente no presentan ningún tipo de trastorno. Además, en el grupo de suspensión de condena los 136 sujetos diagnosticados con trastornos de personalidad presentan una media de 2.66 trastornos por sujeto frente a los 254 sujetos del grupo de prisión con una media de 3.85 trastornos de personalidad por sujeto.

Dentro de las escalas básicas de trastornos de personalidad, el trastorno más frecuente es el compulsivo con 82 y 51 sujetos (suspensión de condena y grupo de prisión respectivamente), lo que supone un $59.9 \%$ y $63.7 \%$ sobre el total de cada submuestra. A continuación, los trastornos más frecuentes por orden de prevalencia en ambos grupos son el dependiente, narcisista y antisocial.

Nuevamente, y dentro de las escalas de personalidad patológica más severa, aparecen por orden de prevalencia el trastorno paranoide y el trastorno esquizotípico con un $24.1 \%$ y $19.7 \%$ respectivamente sobre el total de la muestra de suspensión de condena, y un $40 \%$ y $35 \%$ respectivamente en la muestra de prisión.

Cuando se compara la frecuencia de los trastornos hallados en cada uno de los grupos (suspensión de condena y grupo de prisión), se encuentran diferencias estadísticamente significativas en varias escalas. Así, en el grupo de prisión aparece una tasa superior en trastornos de personalidad histriónica, dependiente, agresivo-sádica, y sobre todo más importante, en las escalas de personalidad patológica más severa, esquizotípica, límite y paranoide frente al grupo de suspensión de condena.

\section{Conclusiones}

En este estudio se ha llevado a cabo un análisis de las dimensiones y trastornos de personalidad que presenta una muestra amplia de maltratadores a la pareja. Asimismo, se han comparado los resultados obtenidos en las variables de personalidad entre un grupo de agresores condenados a una pena de prisión por haber cometido un delito de violencia de género con los obtenidos por otro grupo de agresores a los que se les ha aplicado una suspensión de la ejecución de la pena, a cambio de participar de un programa de tratamiento específico para la violencia de género.
Los resultados obtenidos muestran la presencia de una alta tasa de trastornos de personalidad entre los agresores estudiados. En concreto, en este estudio el $79.3 \%$ de los sujetos de la muestra presenta un trastorno de personalidad (casi 8 de cada de 10). Esta cifra es sin duda muy elevada, pero coincide con las obtenidas en otros estudios previos desarrollados tanto en nuestro país (Fernández-Montalvo y Echeburúa, 2008), como en el ámbito internacional (Hart, et al., 1993). En el estudio de FernándezMontalvo y Echeburúa (2008), por ejemplo, con una muestra de 76 hombres condenados a una pena de prisión por un delito de violencia grave contra la pareja, el $86,8 \%$ presentaba al menos un trastorno de personalidad según el MCMI-II. Esta cifra es similar a la obtenida en este estudio por la submuestra de agresores en prisión (el $82.5 \%$ de los mismos presentaba un trastorno de personalidad). En el estudio de Hart et al. (1997), el 80\% de los agresores estudiados presentaba un trastorno de personalidad.

Sin embargo, estas cifras tan altas se relacionan probablemente con la tendencia observada en los autoinformes en general, y en el MCMI-II en particular, a sobrediagnosticar los casos clínicos. De hecho, en la revisión llevada a cabo por FernándezMontalvo y Echeburúa (2006), en la que se comparan los datos obtenidos con distintos instrumentos de evaluación, se pone de manifiesto la tendencia del MCMI-II a sobrediagnosticar los trastornos de personalidad en comparación con las entrevistas clínicas específicas, incluso cuando se utiliza una misma muestra clínica. En este sentido, cuando se utiliza el MCMI-III -versión del instrumento más refinada y actualizada para la evaluación de trastornos de personalidad, pero de la que se carecía en España en el momento de evaluar a los pacientes de este estudio-, las tasas obtenidas son sensiblemente más bajas. Así, por ejemplo, Gondolf (1999), utilizando el MCMI-III, obtuvo una tasa del $48 \%$ de trastornos de personalidad en una muestra de 840 maltratadores domésticos.

En cualquier caso, al margen de las dificultades evidentes en la evaluación precisa de estos trastornos, los trastornos de personalidad que aparecen con mayor frecuencia en este estudio (obsesivo-compulsivo, paranoide y dependiente) coinciden con los resultados de otro estudio previo con el mismo ins- 
trumento, pero con muestras distintas (FernándezMontalvo y Echeburúa, 2008). Ello refleja una cierta consistencia en los resultados obtenidos.

Por lo que se refiere a la comparación de ambas submuestras, los sujetos que se encuentra en prisión presentan un perfil de personalidad más grave (mayor puntuación en todas las escalas del MCMIII) que los agresores que participan en los programas de suspensión de condena. La gravedad de los trastornos de personalidad parece estar relacionada con la mayor gravedad del maltrato y, por tanto, con el ingreso en prisión. Parece necesario, por tanto, tener en cuenta la presencia de alteraciones de personalidad en la evaluación clínica de los maltratadores, identificar subtipos específicos de agresores y desarrollar programas de evaluación e intervención adaptados a las características específicas que presentan los agresores que cumplen condena por violencia de género (Ross y Babcock, 2009).

Por otra parte, el análisis de los trastornos de personalidad concretos más prevalentes en la muestra pone de manifiesto la importancia que tiene el trastorno compulsivo de la personalidad en ambos grupos. Como se ha señalado anteriormente, la relevancia de este trastorno en agresores a la pareja se ha puesto ya de manifiesto en estudios previos con el MCMI-II (Fernández-Montalvo y Echeburúa, 2008). Las características de este trastorno de personalidad se relacionan con la presencia de una conducta muy controlada y perfeccionista. Se trata de personas hiperexigentes en su entorno cercano, con gran temor a la desaprobación social y que utilizan muchos mecanismos de defensa para justificar sus actos o conductas. Asimismo, tienen una gran dependencia externa y manifiestan una gran dificultad para la expresión de los sentimientos. Todas estas características coinciden con las conductas observadas en los hombres maltratadores que acuden a consulta. Por tanto, se abre aquí una línea de estudio para el futuro. No obstante, este trastorno no aparece de forma tan relevante en muestras anglosajonas, por lo que las limitaciones del instrumento de evaluación o la influencia cultural pueden estar relacionadas con estos resultados.

Además, cuando se comparan las dos submuestras se observan diferencias significativas en algunos trastornos de personalidad concretos. En este sentido, destaca la presencia de diferencias significativas en los trastornos de mayor gravedad (esquizotípico, límite y paranoide), con una mayor presencia en los agresores en prisión. Probablemente algunas características del entorno carcelario, que acrecientan todavía más la suspicacia y los componentes paranoicos debido a una convivencia interna más tensa y agresiva, puedan contribuir a ello. En cualquier caso, se trata de una mera hipótesis que requiere una comprobación empírica.

En suma, los resultados de este estudio muestran una alta tasa de trastornos de personalidad en los hombres maltratadores en general, y mucho más marcada aún en aquellos que se encuentran en prisión. Ello indica la necesidad de continuar con esta línea de investigación, de cara a establecer posibles subtipos de agresores que puedan llegar a beneficiarse de protocolos de tratamiento adaptados a sus características individuales. Esta constituye, sin duda, una de las líneas más actuales de investigación en el ámbito de la violencia de género (Ross y Babcock, 2009).

\section{Agradecimientos}

Los autores agradecen al Servicio Social de Justicia del Gobierno de Navarra las facilidades y ayuda proporcionada para la elaboración de esta investigación.

\section{Referencias}

Boira, S. y Jodrá, P. (2010). Psicopatología, características de la violencia y abandonos en programas para hombres violentos con la pareja: resultados en un dispositivo de intervención. Psicothema, 22, 593-599.

Dinwiddie, S. (1992). Psychiatric disorders among wife batterers. Comprehensive Psychiatry, 33, 411-416.

Echeburúa, E. y Fernández-Montalvo, J. (2007). Male batterers with and without psychopathy: An exploratory study in Spanish prisons. International Journal of Offender Therapy and Comparative Criminology, 51, 254-263. 
Echeburúa, E., Fernández-Montalvo, J. y Amor, P. J. (2003). Psychopathological profile of men convicted of gender violence: A study in the prisons of Spain. Journal of Interpersonal Violence, 18, 798-812.

Fernández-Montalvo, J. (2009). La violencia de género y los trastornos de personalidad. Jano. Medicina y Humanidades, 1752, 21-23.

Fernández-Montalvo, J. y Echeburúa, E. (1997). Variables psicopatológicas y distorsiones cognitivas de los maltratadores en el hogar: un análisis descriptivo. Análisis y Modificación de Conducta, 23, 151-180.

Fernández-Montalvo, J. y Echeburúa, E. (2005). Hombres condenados por violencia grave contra la pareja: un estudio psicopatológico. Análisis y Modificación de Conducta, 31, 451-475.

Fernández-Montalvo, J. y Echeburúa, E. (2006). Uso y abuso de los autoinformes en la evaluación de los trastornos de personalidad. Revista de Psicopatología y Psicología Clínica, 11, 1-12.

Fernández-Montalvo, J. y Echeburúa, E. (2008). Trastornos de personalidad y psicopatía en hombres condenados por violencia grave contra la pareja: un estudio en las cárceles españolas. Psicothema, 20, 193-198.

Fernández-Montalvo, J., Echeburúa, E. y Amor, P. J. (2005). Aggressors against women in prison and in the community: An exploratory study of a differential profile. International Journal of Offender Therapy and Comparative Criminology, 49, 158-167.

Gondolf, E. W. (1999). MCMI-III results for batterer program participants in four cities: Less "pathological" than expected. Journal of Family Violence, 14, 1-17.

Hamberger, L. K. y Hastings, J. E. (1991). Personality correlates of men who batter and nonviolent men: Some continuities and discontinuities. Journal of Family Violence, 6, 131-148.

Hare, R. D. (2001). Psychopaths and their nature. In A. Raine y J. Sanmartín (Eds.), Violence and psy- chopathy (pp. 5-34). New York: Kluwer Academic/Plenum Publishers.

Hart, S. D., Dutton, D. G. y Newlove, T. (1993). The prevalence of personality disorder amongst wife assaulters. Journal of Personality Disorders, 7, 329-341.

Huss, M. T. y Langhinrichsen-Rohling, J. (2000). Identification of the psychopathic batterer: The clinical, legal, and policy implications. Aggression and Violent Behavior, 5, 403-422.

Koeter, M. W. J. y Hartgers, C. (1997). European addiction severity index europasi. Cost a6. Preliminary procedure for the computation of the europasi composite scores. The Amsterdam Institute for Addiction Research.

Millon, T. (1997). Millon Clinical Multiaxial Inventory- II (MCMI II). Minneapolis: National Computer Systems.

Nicholls, T. L., Roesch, R., Olley, M. C., Ogloff, J. R. y Hemphill, J. F. (2005). Jail Screening Assessment Tool (JSAT): Guidelines for Mental Health Screening in Jails. Vancouver: Mental Health, Law, and Policy Institute.

Quinsey, V. L., Harris, G. T., Rice, M. E. y Cormier, C. A. (1998). Violent Offenders. Appraising and Managing Risk. Washington: American Psychological Association.

Redondo, N., Graña, J. L. y González, L. (2009). Características sociodemográficas y delictivas de maltratradores en tratamiento psicológico. Psicopatología Clínica, Legal y Forense, 9, 49-61.

Ross, J. M. y Babcock, J. C. (2009). Proactive and reactive violence among intimate partner violent men diagnosed with antisocial and borderline personality disorder. Journal of Family Violence, 24, 607-617.

Weltzer, S. J. (1990). The Millon Clinical Multiaxial Inventory (MCMI): A review. Journal of Personality Assessment, 55, 445-464.

White, R. J. y Gondolf, E. W. (2000). Implications of Personality Profiles for Batterer Treatment. Journal of Interpersonal Violence, 15, 467-488.

Manuscrito recibido:20/01/2011

Revisión recibida: 22/02/2011

Aceptado: 25/03/2011 